\title{
A Distributed Intelligent System for Emergency Convoy
}

\author{
M. Benalla, B. Achchab, H. Hrimech

\begin{abstract}
Univ. Hassan 1, Higher School of Technology, Laboratory of Analysis and Modeling Systems for Decision Support, Berrechid, Morocco
\end{abstract}

\begin{abstract}
The general problem that guides this research is the ability to design a distributed intelligent system for guiding the emergency convoys; a solution that will be based on a group of agents and on the analysis of traffic in order to generate collective functional response. It fits into the broader issue of Distributed Artificial System (DAI), which is to operate a cooperatively computer agent into multi-agents system (MAS). This article describes conceptually two fundamental questions of emergency convoys. The first question is dedicated to find a response to the traffic situation (i.e. fluid way), while the second is devoted to the convoy orientation; while putting the point on the distributed and cooperative resolution for the general problem.
\end{abstract}

Keyword - Traffic Flow, Multi Agent System, Shortest Path, Iot, Graph Theory.

\section{INTRODUCTION}

$\mathrm{T}$ HE Distributed artificial Intelligence had its inauguration in the late 1970s [1]. The definition, in its most common acceptance of research of artificial intelligence is the study and design of artificial agents organization for intelligent systems [2]. DAI has emerged specifically to develop and solve problems often encountered in everyday life and which require to lend them a collective intelligence. Existing works consist essentially on predictive simulations (e.g. traffic flow) and on research in metaheuristic optimization (e.g. swarm intelligence) and so on.

The emergency convoy problem is a large issue, an issue centered optimization, so the arrival of the convoy to its destination in a reasonable time, is one of the main factors that reduce damage. The convoy guidance from the source to the target in the shortest time and in a safety environment is challenge that takes in its implication the following interrelated aspects: traffic density, road signs, and roads infrastructure quality.

For this purpose, the taking into account of these three aspects constitutes a basic foundation of emergency convoy problem. Traffic density which reflects the state of the environmental circulation leads a movement resistance of the convoy, more that this density is high, the friction to move increases in parallel direction. Sometimes, traffic situation reaches a case where vehicles remain motionless during seconds; thus, representing the worst case of a convoy and certainly not a wishing state. Road signs are also one of the formative elements of traffic density level, by means of their predetermined rules process the vehicles as the same level without considered priority cases of traffic (e.g. convoy of prisoners). While the quality of infrastructure plays a vital role for credible and fluid passage of emergency convoys. For this introduction, the next section makes clear the problem of emergency convoy.

\section{The Emergency Convoy Problem}

Nowadays, the emergency convoy confronts difficulties to reach the destination because of the complex configuration of road traffic network. Various road users continuously adjust their actions to avoid collisions in order to get into the desired environment. This problem becomes much serious when heterogeneous goals of road users meet; the thing that creates traffic jams and from time to time accidents, frequently trick to surpass by an emergency convoy. The distributed artificial intelligence is the driving force behind the resolution of this type of phenomenon which is inherently distributed. It provides a distributed resolution mechanisms through the focus on the communication and cooperation principle. For [3] the faster response, increased flexibility, robustness, resource sharing, graceful degradation and better adaptability are all advantages taking place into concept (i.e. DAI). In addition, with the emergence of the internet of things (IoT) paradigm, the development of autonomous entities becomes a promoter tool since the (IoT) exploits the internet network for information exchange.

This problem can be seen from two perspectives; a traffic manager's point of view, and an emergency conveyor's point of view: for the emergency conveyor's view are only problems to arrive safety and in a reasonable way to the destination; for the traffic manager's view it is to ensure control of the traffic flow.

The urban traffic system is composed of a network, operating rules, management and control system, and entities taking the network. The network is a set of communications and intersection axes [4]. This latter is in perpetually dynamic, which raises the complexity level. The paper will consider the problem of guiding emergency convoy from its departure point to the target, while using the shortest path with an easy passage (i.e. fluid traffic).

In the classical model of emergency convoy routing, conveyors exploit their knowledge about traffic situations, constantly watching the environment state and necessarily react to achieve the goal. So the anticipation field is limited to human feelings, particularly on the look.

\section{RELATED WORKS}

Existing studies do not address the emergency convoy problem itself, but the problem separately, There are those who are interested in the scope of road convoy problems (i.e. travel in platoon) as [5] [6] [7], while others put the points on the optimization problem of shortest path as [8] [9][15].

\section{An Agent- Based Model for Traffic Fluidization for EMERGENCY CONVOY}

\section{A. The notion of agent}

There is a large number of works on the agents, the definitions 
of agents and multi-agent systems. According to [10] the author, he considers the agent as a physical or abstract entity capable of acting on itself and on its environment, having a partial representation thereof that can communicate with other agents and whose behavior is the result of his observations, his knowledge and interactions with other agents.

Nowadays, a number of more and more software systems are designed in terms of agents and multi-agent systems in which software components rather act as independent individual entities, notably agents, instead of being only the components of the system. In problems of road traffic, this can be a vehicle, traffic lights, crossing, and so on.

\section{B. Agent and traffic fluidization}

The guiding complexity of emergency convoy is subjected to two levels, the first level focuses on finding the shortest paths, and the second level aims to improve traffic flow. The agent model for traffic fluidization in this paper puts back on the hierarchical architecture of information systems architecture that consists of three levels: a high level of decision, an intermediate level responsible for communication and coordination, and a low level for execution.

The hierarchical form has a set of privileges in an agent-based configuration. It allows in one hand to reduce redundancy and the amount of information exchanged between levels. On the other hand, to limit the number of communications between agents, and makes direct scheduling instructions.

The principal agents that coexist in the proposed model are the first and the last vehicle of emergency convoy, traffic lights, crossroads, coordinators, and the shortest path. All agents endow computational capabilities and communications models. A general modeling of model is given in Fig. 1.

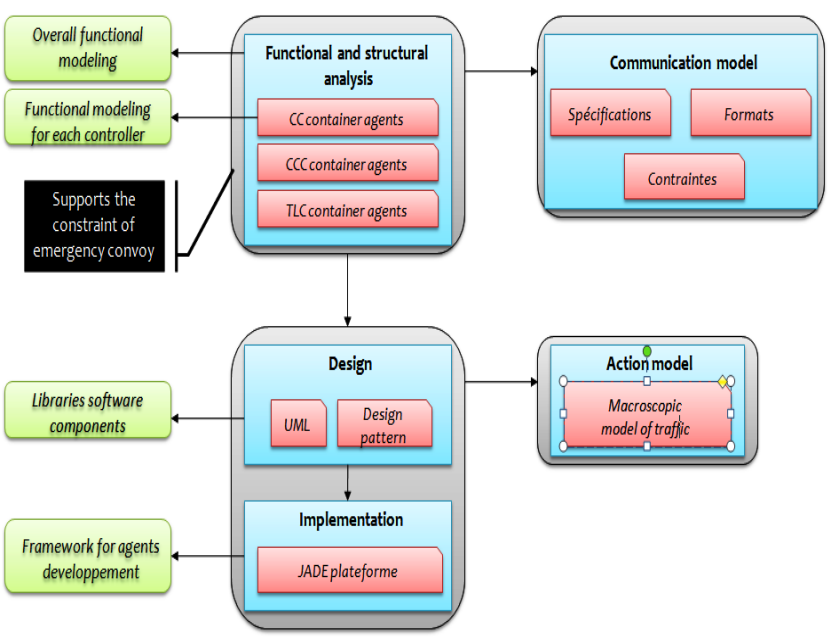

Fig. 1. A general modeling of the model

The communication model is a key factor in the effectiveness of the system, as we speak of a society of agents. It describes the communication forms between the model structures (i.e. CC, TLC, and $\mathrm{CCC}$ ). These structures also containers of the above agents will undergo a definition in section $\mathrm{V}$. The communication model was evoked to ensure system integrality and to ensure the autonomy of structures as well.

In the model, the traffic lights in the intersection and in the singleton are the decision point to solve the traffic fluidization problem. Generally, traffic lights are in two modes, a mode for vehicles (i.e. light red, orange and green) and a mode for pedestrians (i.e. red and green lights). Before proceeding to the description of the traffic fluidization components model of emergency convoy which is based on a group of agents in cooperation, it is necessary to highlight the strategies used to control traffic lights in the intersections since it is the decision point for the model as described above.

In the literature, there are three types of strategies [11], each strategy has its own rules to follow. The control variables of traffic lights are: the length of the cycle; the length of "active time"; the point of time when the cycle starts.

\section{a) Strategy 1: the semiactuated regime}

This strategy is used when the traffic flow in a route gets to an intersection which is considered important in term of vehicle flow. Then the traffic light remains green until the other road (i.e. not busy) marks the approach of vehicles. Note that the busy road (i.e. green light), the light cycle is predetermined to a maximum time even if the unoccupied road does not mark the presence of vehicles.

\section{b) Strategy 2: the platooning regime}

It is a technique exploited in moderate traffic flow. The vehicles travel in platoon separated by distances (i.e. time interval) facilitates the task to the control system of urban traffic. The traffic light is green when vehicles are approaching and red when a distance appears. It is a recommended strategy when it is possible to apply it.

c) Strategy 3: the regime to control individual characteristics separately

This mode of operation is based on three sets of rules which are ranked in the order of usage priority: modify cycle length; change cycle splits; change cycle start time. Each control has different variables

According to these strategies and as this is the issue of emergency convoys, an emergency strategy must take place, dealing with the emergency phenomenon in its entirety. From this point of view, the emergency strategy will help in emergency cases (e.g. ambulances, prisoner convoy, firefighter convoy, gear trains and so on) to put the traffic light in green mode as soon as the emergence of the convoy and in red mode every traffic light of the supervised environment by the Central Controller (CC) (i.e. crossroads lights, pedestrians lights).

\section{A Distributed InTELligent SySTem FOR EMERGENCY Convoy (SDICU)}

Through the multi-agent definition of our hierarchical system and a general modeling in Fig. 1, a distributed system for emergency convoys is designed. The general scheme consists of three hierarchical levels, Fig. 2: the Central Controller (decision-maker); the Communication and Coordination Controller (information provider); the Traffic Lights Controller (executor decision).

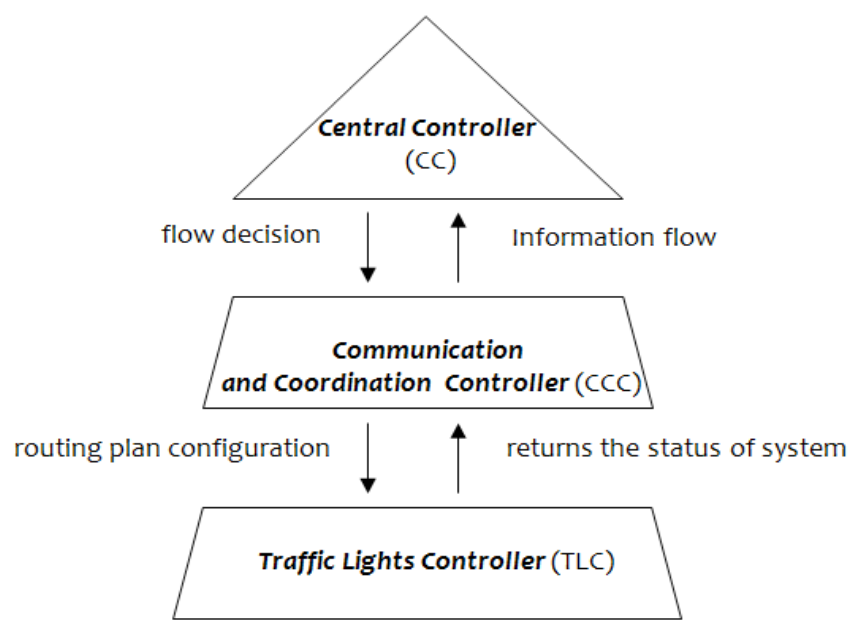

Fig. 2. levels of the multi-agent environment 
As shown in Fig. 2, the (SDICU) consists of the functional components, Fig. 3. The central controller as the name indicates, establishes a policy decision on road map taking into account the information required by the subject (i.e. the communication and coordination controller); because the color of the traffic lights depends on its control strategy (i.e. emergency strategy). While the communication and coordination controller (CCC) reflects all the operations that will be applied on the traffic lights controller, based on the decisions flow provided by the (CC). In addition, the (CCC) is responsible for collecting information about the environment in which it is located. The (TLC) remains an application agent of orders received by the (CCC).

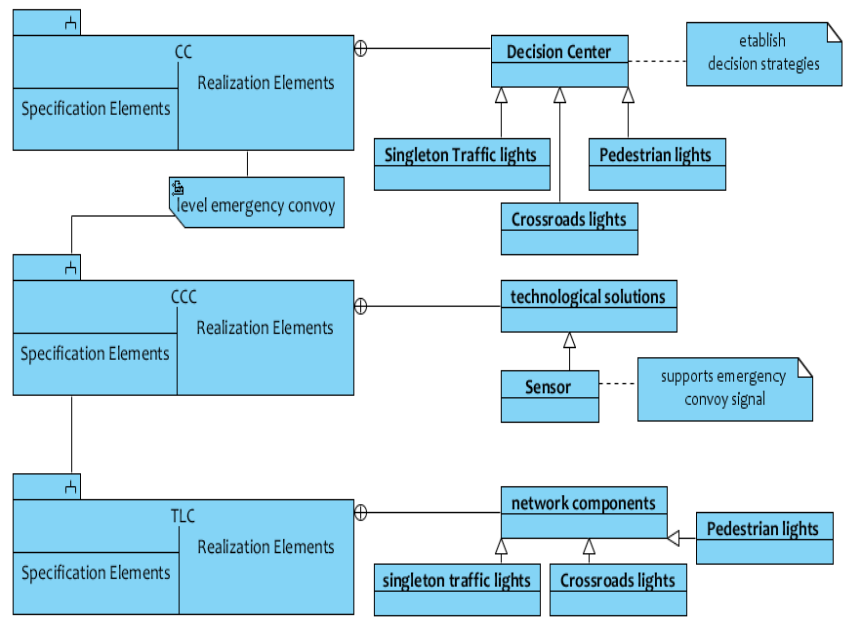

Fig. 3. Some functional components of environment

\section{A. Finding the shortest paths}

The orientation of emergency convoys is a complex challenge, regarding the multitude of ways to be followed. The emergency convoy first tries to reach the destination by travelling a short distance. It is mentioned that among agents that coexist in the system, an agent is in charge of calculating the shortest path.

The itinerary choice by an emergency convoy is governed by a prejudiced law on its capability in real time or by a stochastic law determining the probability of a point that will be visited by an exploration process. From a graph theory viewpoint, the problem can be formulated as follows: a graph $\mathrm{G}$ exposing all itineraries which emergency convoy can take; a set of end points (i.e. intersection) representing one or more issues on an edge (i.e. road segment). An edge is a combination linking each two points. In other words, let $G=(V, E)$ be a complete graph with a collection of nodes $V=\left(v_{0}, v_{1}, v_{2}, \ldots, v_{n}\right)$ and a collection of edges $E=\left(v_{i}, v_{j}\right): v_{i}, v_{j} \in V, i \neq j$

In the literature there is a large number of calculation algorithms for the shortest path [12]. The Dijkstra's method, Fig 4, is a well known calculation problem of shortest path. It consists of a weighted directed graph for which all evaluations are positive to find the shortest between a node $v$ and all other nodes. For each node $v$, we designate the distance between $u$ and $v$ by $D[u]$. By convention if there is no path between $u$ and $v$ we have $d(v, u)=\infty$.

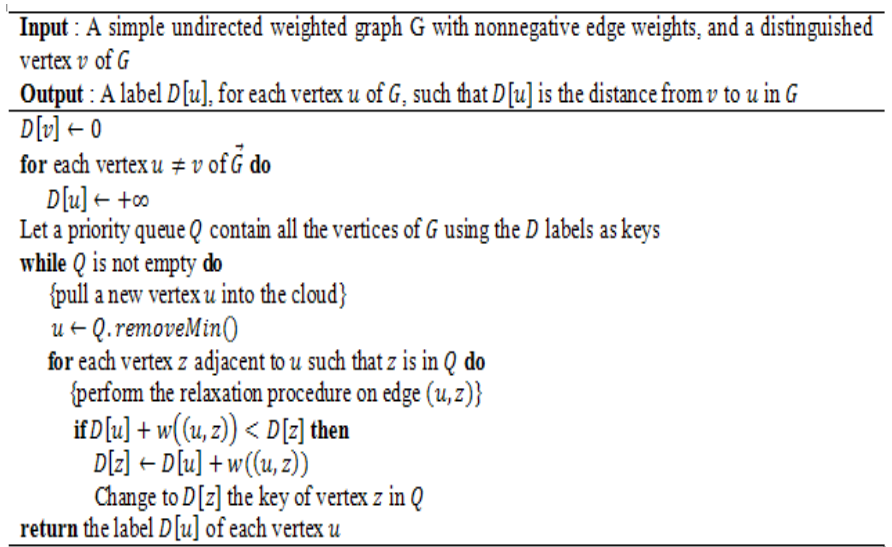

Fig. 4. Dijkstra's algorithm for a graph $G$, starting from a vertex $v$.

\section{B. Functioning}

Initially, the study in this level (research the shortest distance) includes a step process.

1) Determination of the map of the city pointing out all possible routes and available.

2) On the map, the application of a points identification algorithm which will assign different symbols under the following rule:

- For each traffic intersection, assign the symbol "Inter",

- For each traffic intersection with traffic lights, affect the symbol "InterPF",

- For each roundabout, assign the symbol "RPT",

3) Calculation distance in meters between each two points connected,

\section{4) Assignment of random characters for each point,}

5) Build a matrix labeled with these characters. The combinations of values are distances in meters,

\section{6) Running the Dijkstra algorithm for finding the shortest} path.

After this, each emergency convoy will be integrated in a telecommunications network through the Internet of Things concept. At this moment, the shortest path agent communicates with each itinerary point where there are agents controllers responsible for communication and coordination. Each communication and coordination controller agent calculates the estimated time of arrival of the convoy based on their speed which is also provided by the shortest path agent.

At the time of approach of emergency convoy, the agent (CCC) determines the time for the active traffic light to turn green in the convoy sense based on velocity convoy (i.e. advanced preparation of environmental pass) to avoid road collisions. Subsequently, the same agent sends this information to the (CC) agent who charges to apply the aforementioned emergency strategy by returning instructions to the (CCC) agent that communicates them with the controller agent of any traffic light. After the convoy pass (i.e. pass timer), the (CCC) from information collected from its environment (i.e. sensor) requests by forwarding this information to $(\mathrm{CC})$ the traffic strategy to be in place for the supervised area, Fig. 5. 


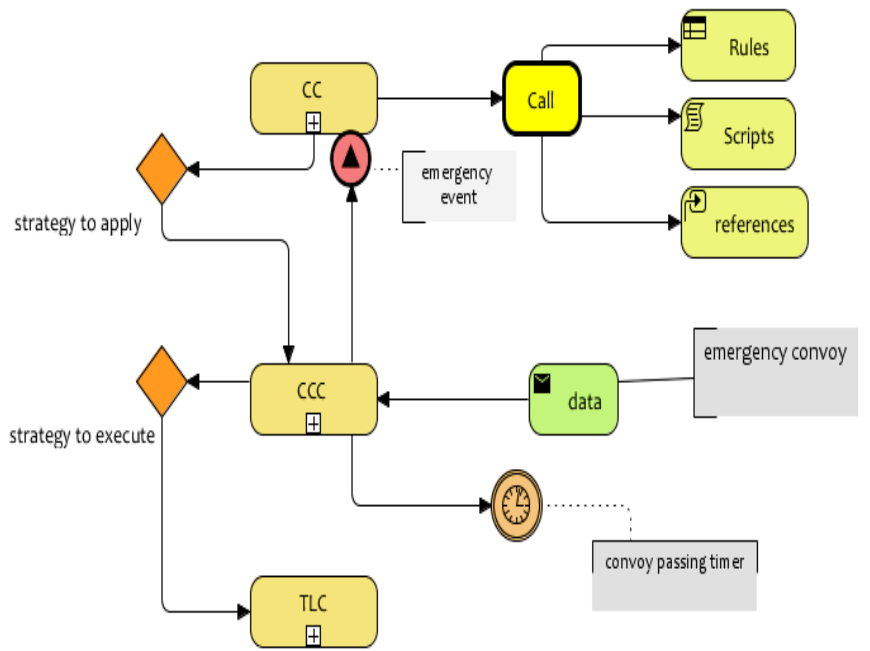

Fig. 5. operating process of the solution

\section{CONCLUSION}

The work presented in this paper is an opening on a number of possibilities. The key of this opening passes undoubtedly by experimental work. The complex configuration of the road network makes it difficult to present a complete solution. In some emergency cases, convoys are face to face where a scheduling problem.

This paper only provides ideas for solving this kind problem of road traffic. We have seen how aspects: distributed artificial intelligence, multi-agent systems are essential to achieve a whole imitating artificial intelligent behavior. We wish to continue for some time our research on the experimental basis. The main features are multi-agent simulation and distributed problem solving, algorithms traffic, implementation of hardware technology; other issues will be raised by prediction but it is not impossible to envisage forthwith transposing a number of our results into reality, which would provide some opportunity to the solution that we defend.

\section{ACKNOWLEDGMENT}

This work is supported by the grant of National Center for Scientific and Technical Research (CNRST- Morocco): No. K005/006 for the first author.

\section{REFERENCES}

[1] G. Weiss, "A modern Approach to Distributed Artificial Intelligence", page 1-2, MIT Press, 1999.

[2] J. Erceau, J. Ferber, "l'Intelligence Artificielle Distribuée, vol. 233

[3] N.V. Findler and G.D. Elder, "Multiagent Coordination and Cooperation in a Distributed Dynamic Environement with Limited Resources", Artificial Intelligence in Engineering, 9(1995), pp 229-238.

[4] M. Chabrol, D. Sarramia, " Modélisation orientée objet du système d'information des Systèmes de Trafic Urbain : une approche multi-agents »

[5] D. Goldstein, T. Shehab, J Casse, Hsiu-Chin Lin, "On the formulation and solution of the convoy routing problem", Transportation Research Part E, 2009.

[6] A. Rasheed, H. Zia, F. Hashmi, U. Hadi, W. Naim, S. Ajmal, "Fleet \& Convoy Management Using VANET", Journal of Computer Networks, 2013, Vol. 1, No 1, 1-9.

[7] V. Baines, J. Padget, "Communication and metrics in agent convoy organization", Dept. of computer science, university of Bath.

[8] Y. Huang, G. Zhang, J. Wang, “An Optimization Dijkstra Algorithm Based on Two-function Limitation Strategy, Image and signal Processing, 2009, $1-4$.

[9] S. Kadry, A. Abdallah, J. Chibli, "On The Optimization of Dijkstra's Algorithm”, Electrical Engineering, 2012, Vol. 2, pp 393-397.

[10] J. Ferber, « Les systèmes multi-agents : vers une intelligence collective », InterEditions, ISBN : 2-72-96-0572-X, 1995.

[11] N.V. Findler, "Contibutions to a Computer-Based Theory of Strategies", Springer-Verlag, 1990.

[12] M.T. Goodrich, R. Tamassia, "Algorithm Design: Foundations, Analysis, and Internet Examples", pp 341-345

[13] M. Li, J. Hallam, L. Pryor, S. Chan, K. Chong, "A Cooperative Intelligent System for Urban Traffic Problems", Proceedings of the 1996 IEEE International Symposium on Intelligent Control Dearbor, 1996.

[14] J.M. Fernandes, E. Oliveira, "TraMas: Traffic Control through Behaviorbased Multi-Agent System", ResearchGate, 2015.

[15] Guillermo Cueva-Fernandez et al., "Fuzzy decision method to improving the information exchange in a vehicle sensor tracking system.", Applied Soft Computing, 35, pp. 708-716, 2015.

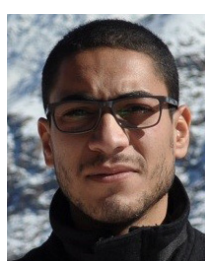

Mohammed Benalla received his B.Sc. in Distributed Information System from Hassan II University. He is a Ph.D. student of the Higher School of Technology at Hassan 1st University. His research interests include distributed artificial intelligence and parallel and distributed computing. His research topic is about adaptation of flow traffic in urban growth during the Ph.D. program.

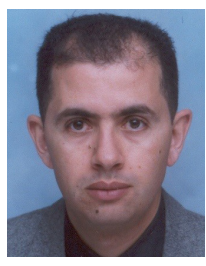

Boujemâa Achchab is a Full Professor in the Computer Science and Mathematics Department of the Higher School of Technology at Hassan 1st University and the director of the Laboratory of Analysis and Modeling Systems for Decision Support. He has a Ph.D. in applied mathematics from the University Lyon 1, France. His research interests include numerical analysis, data mining and econometric modeling.

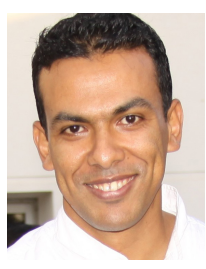

Hamid Hrimech is an assistant Professor in the Computer Science and Mathematics Department of the Higher School of Technology at Hassan 1st University. He has a Ph.D. from Arts et Métiers ParisTech (France) in computer science. His research interests include collaborative interactions in collaborative virtual environment, AI, and driving simulation. 